\title{
EMERGENCIA SOCIOSANITARIA EN CONSUMO DE RIESGO DE ALCOHOL Y SÍNTOMAS DE DEPENDENCIA EN JÓVENES
}

\section{SOCIO HEALTH EMERGENCY RISK OF ALCOHOL CONSUMPTION AND SYMPTOMS OF RELIANCE ON YOUTH}

María de la Villa Moral Jiménez ${ }^{1}$, Carolina Bringas Molleda² ${ }^{2}$ Anastasio Ovejero Bernal ${ }^{3}$, Luz Anyela Morales Quintero ${ }^{4}$, Francisco Javier Rodríguez Díaz ${ }^{1}$

${ }^{1}$ Universidad de Oviedo

¿Universidad de Extremadura

${ }^{3}$ Universidad de Valladolid

${ }^{4}$ Universidad Autónoma de Puebla, México

\section{Abstract}

Introduction. The excess consumption of alcohol in the adolescent and young population constitutes a problem of great magnitude and a health care. Objective. Evaluate the psychometric characteristics of the Alcohol Use Disorders Identification Test (AUDIT) in young people and studying alcohol use disorders (excessive alcohol consumption, harmful and dependent) in adolescents and young Spaniards and Mexicans, establishing a differential analysis. Method. Descriptive observational study with cross-sectional analysis of 1313 adolescents and youth of Spain and Mexico, with aged between 13 and 19 years, and both sexes. It has chosen a design correlational no experimental research, conducted on a within-subject design and selecting participants. Results. Have confirmed the psychometric properties of the test sample AUDIT juvenile adequate construct validity and reliability, and its factorial structure. In assessing disorders alcohol consumption, confirms the existence of significant differences between young Spaniards and Mexicans, all indicators tested, resulting in a size large effect. Conclusions. Intensive alcohol consumption in the youth group constitutes a problem with many implications, hence the importance of assessing risk behaviors and symptoms of dependence in young people.

Keywords: alcohol, risk underage drinking, binge drinking, high school and college students.

\section{Resumen}

Introducción. El exceso de consumo de alcohol en la población adolescente y joven constituye un problema de gran magnitud y una emergencia sociosanitaria. Objetivo. Evaluar las características psicométricas del Alcohol Use Disorders Identification Test (AUDIT) en población juvenil y estudiar los trastornos por consumo de alcohol en adolescentes y jóvenes españoles y mexicanos, estableciéndose un análisis diferencial. Metodología. Estudio observacional descriptivo con análisis transversal de 1313 adolescentes y jóvenes de España y de México, con edades comprendidas entre los 13 y los 19 años y de ambos sexos. Se ha optado por un diseño de investigación no experimental correlacional, realizado sobre un diseño intrasujeto y seleccionando a los participantes mediante un muestreo probabilístico. Resultados. Se han confirmado las propiedades psicométricas del test AUDIT en muestra juvenil con una adecuada validez de constructo y confiabilidad y su estructura factorial. Asimismo, se confirma la existencia de diferencias significativas entre jóvenes españoles y mexicanos, en todos los indicadores evaluados, obteniéndose un tamaño de efecto elevado. Conclusiones. El consumo intensivo de alcohol en el colectivo juvenil constituye una problemática con múltiples implicaciones, de ahí la importancia de evaluar las conductas de riesgo y los síntomas de dependencia en jóvenes.

Palabras clave: alcohol, consumo de riesgo de alcohol, atracón, estudiantes de secundaria y universitarios.

Correspondencia: Francisco Javier Rodríguez Díaz

gallego@uniovi.es 
El consumo abusivo de alcohol en población infantojuvenil constituye un problema de gran magnitud y una auténtica emergencia sociosanitaria. Específicamente, el Consumo Intensivo de Alcohol (CIA) corresponde a una ingesta de 60 gramos o más en varones y 40 gramos o más en mujeres, durante un intervalo de 2-3 horas (Motos, Cortés, Giménez, \& Cadaveira, 2015). A partir de la modificación del patrón de consumo de alcohol de tipo mediterráneo por modalidades de tipo anglosajón (binge drinking) se han incrementado los riesgos sobre la salud física y psíquica de estos consumidores (Luque, Gómez, Cortés, Espejo, \& Giménez, 2014). Este patrón binge drinking o atracón se caracteriza por representar una modalidad de consumo con mayor incidencia en la población juvenil, con un manifiesto descenso de la edad de los bebedores abusivos, con igualación de consumo por sexos y asociado a una baja percepción de riesgo (Balodis, Potenza, \& Olmstead, 2009; Carlson, Johnson, \& Jacobs, 2009).

Semejante problemática presenta múltiples implicaciones no solo a nivel individual, sino también familiar, social y comunitario. A nivel global, al valorar las complicaciones relacionadas con el alcoholismo se estima que representa de entre el $2 \%$ al $9 \%$ de las consultas en atención primaria (Villar, Pinto, Barizon, \& Ferreira, 2016). Además, el consumo abusivo de alcohol se asocia a altos índices de morbilidad y mortalidad y genera importantes daños en la esfera psicosocial (Guitart et al., 2011; Moral, \& Ovejero, 2011; Morales, Bringas, Moral, Rodríguez, Cetz, \& Arriaga, 2015). También deriva en serios problemas de tipo físiopatológico así como urgencias en lesionados de tráfico con alcoholemia positiva, por síndromes de dependencia alcohólica, por intoxicaciones agudas atendidas en urgencias que representan casi el $1 \%$ de las asistencias en los servicios de urgencias hospitalarios (SUH) y se asocia a diversas intervenciones de urgencias por consumo abusivo de alcohol y otras drogas de abuso emergentes (Guitart et al., 2011; Clemente, Echarte, Aguirre, Puente, Iglesias, \& Supervía, 2011; Galicia, Alonso, \& Nogué, 2014). En concreto, en España, el CIA en jóvenes se asocia con policonsumo y representa un factor de riesgo para el desarrollo posterior de abuso/dependencia alcohólica. Asimismo, del consumo intensivo juvenil de alcohol se derivan, aparte de síntomas de intoxicación, conflictos interpersonales, conductas sexuales de riesgo, comportamientos agresivos, lesiones de diverso tipo, así como conductas infractoras, entre otras (Isorna, Fariña, Sierra, \& Vallejo, 2015). Respecto a las urgencias hospitalarias relacionadas con el alcohol en jóvenes, en España son pocos los estudios realizados, si bien se detecta una clara presencia de las urgencias en los fines de semana o festivos, ${ }^{y}$ una mayor gravedad entre los jóvenes mayores, respecto a los adolescentes, en urgencias entre semana con mayor porcentaje de ingresos hospitalarios (Sánchez, Redondo, García, \& Velázquez, 2012; Sanz, Arias, Trenchs, Curcoy, Matalí, \& Luaces, 2009).

Dado nuestro interés investigador en contrastar dos realidades de consumo juvenil de alcohol, la española y la mexicana, se apunta que en España la problemática de las embriagueces de los jóvenes es considerable, de modo que, de acuerdo con los resultados que se manejan provenientes de la Encuesta sobre Uso de Drogas en Estudiantes de Enseñanzas Secundarias (ESTUDES) 2012/2013 (Delegación del Gobierno para el Plan Nacional sobre Drogas, 2014), se ha hallado que seis de cada diez estudiantes con edades comprendidas entre 14 y 18 años se han emborrachado alguna vez, tres de los cuales lo hicieron en el último mes. Así, según datos de la Encuesta Domiciliaria sobre Alcohol y otras Drogas (EDADES) 2013/2014, el alcohol está presente en el 95\% de los policonsumos; se constata que sigue siendo la sustancia psicoactiva más consumida y registra un ligero incremento. En el grupo de 15 a 17 años dos de cada diez adolescentes se han emborrachado en el último mes y tres de cada diez $(31,9 \%)$ tienen un consumo de riesgo los fines de semana. Respecto al patrón binge drinking, asciende ligeramente el consumo de alcohol con esta modalidad $(15,5 \%$ de los encuestados ha consumido alcohol en forma de atracón en los últimos 30 días frente al $15,2 \%$ en $2011 / 12$ ) y se incrementa con la edad, ya que el $43 \%$ de los estudiantes de 16 años y la mitad de los de 17 se han atracado de alcohol alguna vez en el último mes (Delegación del Gobierno para el Plan Nacional sobre Drogas, 2015).

En contextos latinoamericanos se constatan altas prevalencias de consumo de alcohol, específicamente aplicables al colectivo juvenil. En concreto, en el estudio realizado con estudiantes universitarios mexicanos, aplicando la Prueba de Identificación de Trastornos Derivados del Consumo de Alcohol (AUDIT) (Organización Mundial de la Salud, 1992), se encontró que la prevalencia de consumo de riesgo y dañino de alcohol (con puntaje 
de seis o más en el AUDIT), fue de $18,4 \%$ en el total de la muestra (11,3\% en mujeres y $27,4 \%$ en hombres) en el último año (Díaz, Díaz, Hernández-Ávila, Narro, Fernández, \& Solís, 2008). Al igual que en España, el patrón de consumo juvenil más frecuente en México es el descrito con consumo de altas cantidades por ocasión, constatándose que el $23 \%$ de las personas que consumían alcohol lo habían hecho en los últimos 30 días y que habían llegado a la embriaguez al menos una vez a la semana (29\%). Asimismo, es reseñable que el alcohol fue la sustancia más frecuentemente relacionada con defunciones por accidente (83\%), homicidios (72,2\%), suicidio $(77,6 \%)$ e incluso en las muertes no violentas (73\%) (Secretaría de Salud, Subsecretaría de Prevención y Promoción de la Salud y Dirección General de Epidemiología, 2013).

En definitiva, embriagarse se ha convertido no una consecuencia de un consumo abusivo, sino en la búsqueda de un estado de borrachera que se normaliza en momentos de ocio vinculados al peer group como elemento definitorio de una cultura recreativa juvenil (Luque, Gómez, Cortés, Espejo, \& Giménez, 2014). Semejantes atracones se asocian a una baja percepción de riesgo con distorsión de las consecuencias asociadas a nivel físico, psíquico y psicosocial, lo que permite plantear como objetivo: evaluar las características psicométricas del Alcohol Use Disorders Identification Test (AUDIT) en población juvenil y estudiar los trastornos por consumo de alcohol (consumo de alcohol excesivo, perjudicial y dependiente) en adolescentes y jóvenes españoles y mexicanos, estableciéndose un análisis diferencial.

\section{MÉTODO}

\section{Participantes}

Estudio observacional descriptivo de una serie de casos con análisis transversal realizado en la Universidad de Oviedo (España) y en la Universidad Autónoma de Puebla (México) con estudiantes de Educación Secundaria, Bachillerato y universitarios de Ciencias Sociales y de la Salud. El estudio fue aprobado por la Comisión de Ética de ambos Centros y se realizó siguiendo los principios éticos de la declaración de Helsinki. Para este estudio se ha contado con la colaboración, con consentimiento informado, de 1313 adolescentes y jóvenes de España $(n=520)$ y de México $(n=793)$. Han sido seleccionados mediante un muestreo opinático, en función del cumplimiento de una serie de criterios de inclusión y de la accesibilidad a la muestra, con edades comprendidas entre los 13 y los 19 años $(X=16.02, D T=1.654)$ y de ambos sexos, siendo varones el $38.3 \%(N=503)$ y mujeres, el $61.7 \%(N=810)$.

\section{Variables investigadas e instrumentos de evaluación}

Dado nuestro objetivo de investigación, en este estudio se ha empleado el Test AUDIT, desarrollado para identificar consumos de riesgo y perjudiciales, así como sugerir dependencia de alcohol (Saunders, Aasland, Babor, De la Fuente, \& Grant, 1993). Es una prueba de tamizaje integrada por diez reactivos, de los cuales los tres primeros se centran en analizar la cantidad y la frecuencia de consumo y en los restantes se exploran los potenciales trastornos causados por los consumos perjudiciales. Dada su caracterización de riesgo, de dependencia y consumo perjudicial de alcohol, es consistente con el patrón descrito en el CIE-10 y ha demostrado su eficacia para discriminar adolescentes en riesgo de consumo de alcohol. Por lo tanto, el consumo de riesgo queda definido como un patrón de consumo de alcohol que aumenta el riesgo de consecuencias adversas para el bebedor o para los demás (Organización Mundial de la Salud, 1992). El AUDIT es un instrumento con validez transcultural que ha sido validado tanto en población española (Arias, Ruiz, Fernández, Pérula, Muriel, \& Márquez, 2005; Contel, Gual, \& Farran, 1999), como mexicana (Facundo, Pedrão, Aguilar, García, \& Almanza, 2007; Gómez-Maqueo, Gómez, Morales, \& Pérez, 2009). La consistencia interna del AUDIT se ha estimado en rangos que van de .75 a .94 (valores alpha) en diferentes poblaciones (Gómez-Maqueo, Gómez, Morales, \& Pérez, 2009).

\section{Procedimiento y análisis de datos}

Se ha optado por un diseño de investigación no experimental correlacional, realizado sobre un diseño intrasujeto y seleccionando a los participantes mediante un muestreo probabilístico. Se explicaron los objetivos de la investigación a los adolescentes y jóvenes que han participado en el estudio y se les administró, durante la unidad horaria de clase, la prueba a realizar por parte de profesionales y auxiliares de investigación entrenados al efecto. Fueron informados de la confidencialidad y el 
anonimato de las respuestas, obteniéndose su consentimiento informado.

Se han utilizado diversas técnicas estadísticas para medir la confiabilidad y consistencia interna de la escala AUDIT mediante análisis factoriales exploratorios y confirmatorios a través del modelo de ecuaciones estructurales, análisis de fiabilidad y correlacionales. Para el análisis factorial confirmatorio hemos tenido en cuenta Ios estadísticos CFI y RMSEA. Asimismo, se ha realizado un contraste de medias, utilizando la T de Student, con el objeto de determinar la existencia de diferencias transculturales significativas entre las diferentes variables estudiadas, calculándose el tamaño de efecto. Para el procesamiento y análisis de los datos se ha empleado el paquete estadístico SPSS $21.0 \bigodot$

\section{RESULTADOS}

El plantear evaluar las características psicométricas del AUDIT en muestra juvenil nos ha llevado a establecer la validez de constructo y confiabilidad del instrumento. Se ha hallado un alto valor del Alpha de Cronbach (.929) para los diez reactivos que lo integran y una población de 1313 sujetos. En concreto, los valores de los factores de la estructura factorial oscilan entre .868 hallado en el Factor 1 Consumo de Riesgo de alcohol y .822 obtenido en el Factor 3 Consumo Perjudicial de alcohol (véase Tabla 1). La validez de estructura interna fue probada con un análisis factorial exploratorio, mediante el método de extracción de análisis de componentes principales con rotación ortogonal, hallándose pesos factoriales en el rango de .847 a .681 (véase Tabla 1).

Tabla 1. Análisis de fiabilidad y estructura factorial del AUDIT (N=1313)

\begin{tabular}{|c|c|c|c|c|}
\hline FACTORES & Ítems AUDIT & Contenido de los ítems & $\begin{array}{l}\text { Alpha de } \\
\text { Cronbach }\end{array}$ & Peso Factorial \\
\hline \multirow[t]{3}{*}{ Consumo de Riesgo de alcohol } & 1 & ¿Con qué frecuencia consume alguna bebida alcohólica? & & .828 \\
\hline & 2 & $\begin{array}{l}\text { ¿Cuántas consumiciones de bebidas alcohólicas suele } \\
\text { realizar en un día de consumo normal? }\end{array}$ & .868 & .721 \\
\hline & 3 & $\begin{array}{l}\text { ¿Con qué frecuencia toma } 6 \text { o más bebidas alcohólicas en } \\
\text { una sola ocasión de consumo? }\end{array}$ & & .837 \\
\hline \multirow[t]{3}{*}{ Síntomas de Dependencia } & 4 & $\begin{array}{l}\text { ¿Con qué frecuencia en el curso del último año ha sido } \\
\text { incapaz de parar de beber una vez que había empezado? }\end{array}$ & & .734 \\
\hline & 5 & $\begin{array}{l}\text { ¿Con qué frecuencia en el curso del último año no pudo } \\
\text { hacer lo que se esperaba de usted porque había bebido? }\end{array}$ & .847 & .847 \\
\hline & 6 & $\begin{array}{l}\text { ¿Con qué frecuencia en el curso del último año ha } \\
\text { necesitado beber en ayunas para recuperarse después de } \\
\text { haber bebido mucho el día anterior? }\end{array}$ & & .823 \\
\hline \multirow[t]{4}{*}{ Consumo Perjudicial de alcohol } & 7 & $\begin{array}{l}\text { ¿Con qué frecuencia en el curso del último año ha tenido } \\
\text { remordimientos o sentimientos de culpa después de } \\
\text { haber bebido? }\end{array}$ & & .803 \\
\hline & 8 & $\begin{array}{l}\text { ¿Con qué frecuencia en el curso del último año no ha } \\
\text { podido recordar lo que sucedió la noche anterior porque } \\
\text { había estado bebiendo? }\end{array}$ & & .774 \\
\hline & 9 & $\begin{array}{l}\text { ¿Usted o alguna otra persona han resultado heridos } \\
\text { porque usted había bebido? }\end{array}$ & .822 & .758 \\
\hline & 10 & $\begin{array}{l}\text { ¿Algún familiar, amigo, médico o profesional sanitario ha } \\
\text { mostrado preocupación por su consumo de bebidas } \\
\text { alcohólicas o le ha sugerido que deje de beber? }\end{array}$ & & .681 \\
\hline
\end{tabular}

Asimismo, se han hallado elevadas correlaciones entre los ítems, lo cual es indicativo de su consistencia interna, tal y como se expone en la Tabla 2.

El análisis factorial confirmatorio muestra que el modelo de los tres factores del AUDIT (Consumo de riesgo de alcohol, Síntomas de dependencia, y Consumo perjudicial de alcohol) para el conjunto de la muestra de ambos países es válido: $\mathrm{CFI}=.986$; RMSEA=.052. Al mismo tiempo, existen correlaciones positivas entre subfactores (Ver Figura 1). 
EMERGENCIA SOCIOSANITARIA EN CONSUMO DE RIESGO DE ALCOHOL Y SÍNTOMAS DE DEPENDENCIA EN JÓVENES

Tabla 2. Correlaciones bivariadas AUDIT ( $N=1313)$

\begin{tabular}{|c|c|c|c|c|c|c|c|c|c|c|}
\hline & AUDIT1 & AUDIT2 & AUDIT3 & AUDIT4 & AUDIT5 & AUDIT6 & AUDIT7 & AUDIT8 & AUDIT9 & AUDIT 10 \\
\hline \multicolumn{11}{|l|}{ AUDIT1 } \\
\hline AUDIT2 & $.651 * \star$ & & & & & & & & & \\
\hline AUDIT3 & $.731 * \star$ & $.680 * *$ & & & & & & & & \\
\hline AUDIT4 & $.541 * \star$ & $.458^{\star *}$ & $.571 * \star$ & & & & & & & \\
\hline AUDIT6 & $.604^{* *}$ & $.497^{\star *}$ & $.619 * *$ & $.572^{\star \star}$ & $.759 * \star$ & & & & & \\
\hline AUDIT7 & $.608^{* \star}$ & $.487^{\star \star}$ & $.608^{* \star}$ & $.531 * \star$ & $.694^{\star \star}$ & $.652^{\star \star}$ & & & & \\
\hline AUDIT8 & $.608^{* *}$ & $.509 * *$ & $.597 * \star$ & $.540 * *$ & $.601 * *$ & $.594^{\star *}$ & $.598^{* *}$ & & & \\
\hline AUDIT9 & $.564^{\star \star}$ & $.449 * *$ & $.563^{\star *}$ & $.480 * \star$ & $.615^{\star *}$ & $.606 * *$ & $.563 * *$ & .545 & & \\
\hline AUDIT10 & $.521 * \star$ & $.411 * \star$ & $.499 * \star$ & $.444^{* \star}$ & $.520 \star \star$ & .491 ** & $.509 * \star$ & $.447 * \star$ & $.550 * *$ & \\
\hline
\end{tabular}



Figura 1. Análisis Factorial Confirmatorio del AUDIT ( $N=1313)$ 
Al evaluar los trastornos por consumo de alcohol, el análisis diferencial confirma la existencia de diferencias significativas entre ambas subpoblaciones, jóvenes españoles y mexicanos, en todos los indicadores evaluados: mayor consumo de riesgo, perjudicial y síntomas de dependencia en los primeros, obteniéndose un tamaño de efecto elevado (T.E > .80) (véase Tabla 4).

Tabla 4. Medias y desviaciones típicas del AUDIT, así como comparación de las medias muestrales

\begin{tabular}{|c|c|c|c|c|c|c|}
\hline \multirow{2}{*}{ Reactivos del AUDIT } & \multicolumn{2}{|c|}{ España } & \multicolumn{2}{|c|}{ México } & \multirow{2}{*}{$\mathrm{t}$} & \multirow{2}{*}{$\begin{array}{l}\text { Tamaño de } \\
\text { efecto }\end{array}$} \\
\hline & $x$ & DT & $x$ & DT & & \\
\hline $\begin{array}{l}\text { AUDIT1 } \\
\text { consumo }\end{array}$ & 2,43 & 1.069 & 1.09 & .895 & $-23.603 * \star \star$ & 1.15 \\
\hline AUDIT2 Cantidad típica & 1.51 & .966 & .56 & .980 & $-17.290 * \star \star$ & .88 \\
\hline $\begin{array}{l}\text { AUDIT3 Frecuencia del } \\
\text { consumo elevado }\end{array}$ & 1.56 & .826 & .52 & .735 & 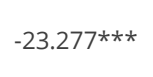 & 1.13 \\
\hline $\begin{array}{l}\text { AUDIT4 Pérdida del control } \\
\text { sobre el consumo }\end{array}$ & 1.35 & .728 & .35 & .839 & $-22.258 * \star *$ & 1.07 \\
\hline $\begin{array}{l}\text { AUDIT5 Aumento de la } \\
\text { relevancia del consumo }\end{array}$ & 1.30 & .645 & .17 & .472 & $-34.483 * * *$ & 1.46 \\
\hline $\begin{array}{l}\text { AUDIT7 Sentimiento de culpa } \\
\text { tras el consumo }\end{array}$ & 1.39 & .668 & .30 & .627 & $-29.461 * * *$ & 1.31 \\
\hline AUDIT8 Lagunas de memoria & 1.53 & .744 & .36 & .872 & $-26.124 * \star \star$ & 1.17 \\
\hline $\begin{array}{l}\text { AUDIT9 Lesiones relacionadas } \\
\text { con el alcohol }\end{array}$ & 1.58 & .854 & .22 & .794 & $-29.119 * \star \star$ & 1.30 \\
\hline $\begin{array}{l}\text { AUDIT10 Otros se preocupan } \\
\text { por el consumo }\end{array}$ & 1.58 & .851 & .41 & 1.101 & $-20.531 * \star \star$ & 1.01 \\
\hline $\begin{array}{l}\text { FACTOR } 1 \text { Consumo de riesgo } \\
\text { de alcohol }\end{array}$ & 1.833 & .830 & .725 & .725 & $-24.879 * \star \star$ & 1.18 \\
\hline $\begin{array}{lcll}\text { FACTOR } 2 & \text { Síntomas de } \\
\text { dependencia } & & \end{array}$ & 1.304 & .540 & .206 & .427 & $-39.009 * \star \star$ & 1.54 \\
\hline $\begin{array}{l}\text { FACTOR } 3 \text { Consumo perjudicial } \\
\text { de alcohol }\end{array}$ & 1.523 & .614 & .324 & .537 & $-36.238 * * *$ & 1.48 \\
\hline
\end{tabular}

\section{DISCUSIÓN}

Dada la gravedad del consumo abusivo de alcohol en la población infanto-juvenil, así como la cantidad de urgencias médicas derivadas del mismo (Carlson, Johnson, \& Jacobs, 2009), parece necesario identificar patrones de consumo en este sector de la población y contar con instrumentos válidos y confiables para este fin. De este modo, este estudio se ha propuesto evaluar las características psicométricas del AUDIT en población juvenil y estudiar los patrones y trastornos de consumo de alcohol en adolescentes y jóvenes españoles y mexicanos.
Con respecto al primer objetivo, nuestros resultados señalan que la confiabilidad interna del AUDIT en el sector de la población estudiada es alto (.929 Alpha de Cronbach), mayor al obtenido en la validación del AUDIT en población general en México, que fue de .87, y mayor también con respecto a otras validaciones realizadas con poblaciones adolescentes donde se obtuvo un coeficiente alpha de .82. En cuanto a los valores de los tres factores en la estructura factorial, nuestros resultados muestran coeficientes altos para cada uno de ellos, con valores entre .822 para el Consumo Perjudicial y .868 para Consumo de Riesgo, mientras en la validación mexicana estos fueron de .87 y .69 respectivamente; en 
el caso de adolescentes los valores han sido superiores a .73 (Medina-Mora, Carreño, \& De la Fuente, 1998; GómezMaqueo, Gómez, Morales, \& Pérez, 2009). Lo anterior demuestra que las escalas resultaron válidas y confiables para ser aplicadas en adolescentes y jóvenes.

Por otro lado, respecto a los patrones y trastornos de consumo de alcohol, el estudio ha confirmado la existencia de diferencias significativas entre los jóvenes españoles y los mexicanos, en todos los indicadores evaluados. Los resultados señalan un patrón de consumo mayor en jóvenes españoles, lo cual es consistente con los datos del Plan Nacional Sobre Drogas -PNSD(Delegación del Gobierno para el Plan Nacional sobre Drogas, 2014), en el que se reconoce un incremento más que considerable de las embriagueces de este sector de la población. De acuerdo con nuestros resultados se confirma una alta frecuencia del patrón de atracón tanto en jóvenes españoles como mexicanos, de modo que por lo que respecta a la frecuencia de consumo el 13,5\% de los jóvenes españoles consume dos o más veces a la semana ( $5,4 \%$ en jóvenes mexicanos) y la frecuencia de consumo elevado medido mediante la frecuencia de consumo de seis o más bebidas alcohólicas en una sola ocasión semanalmente es del 3,3 \% (1,3\% en mexicanos). Respecto a los impactos del consumo perjudicial, el 7,2\% de los jóvenes españoles (6,9 \% de los mexicanos) reconoce que algún profesional sanitario, familiar y/o amigo ha mostrado preocupación por su consumo excesivo. En lo relativo a los síntomas de dependencia, al evaluar la pérdida de control sobre el consumo se ha hallado que el 2,4 de los jóvenes mexicanos reconoce que a diario o casi a diario y el 4,4 de los españoles semanalmente.

Estos datos mantienen una tendencia similar que en estudios previos realizados en España, en donde seis de cada diez estudiantes con edades comprendidas entre 14 y 18 años se han emborrachado alguna vez, y de ellos tres lo hicieron en el último mes. Asimismo, en relación al botellón y las borracheras en España, los resultados de la Encuesta Domiciliaria sobre Alcohol y otras Drogas 2013/2014 indican que tres de cada diez (31,9\%) tiene un consumo de riesgo durante los fines de semana (Delegación del Gobierno para el Plan Nacional sobre Drogas, 2015). En estudios con población mexicana se confirma la alta frecuencia del patrón de consumo consuetudinario (atracón) con una prevalencia del 5.4\%. Así, en los adolescentes el 14,5\% presenta un consumo elevado de alcohol, de modo que en estudiantes universitarios la prevalencia de consumo riesgoso y dañino de alcohol es de 18,4\% en el último año (MedinaMora, Carreño, \& De la Fuente, 1998; Gómez-Maqueo, Gómez, Morales, \& Pérez, 2009), estimándose que en los adolescentes los riesgos de una posible dependencia son aplicables al 4.1\%. Entre las posibles explicaciones de estos resultados deben considerarse las diferencias culturales en cuanto a los contextos en que suele consumirse alcohol y su relación con los procesos de socialización. Mientras en México puede consumirse el alcohol en ocasiones especiales (fiestas específicas), en España representa una pauta de vinculación entre iguales dentro de una cultura juvenil recreativa de fin de semana como cultura de los excesos (Morales, Bringas, Moral, Rodríguez, Cetz, \& Arriaga, 2015; García, Novalbos, Martínez, \& O’Ferrall, 2016).

Como limitaciones de este trabajo contamos con que el proceso de selección muestral no es aleatorio. Los participantes son estudiantes de Educación Secundaria, Bachillerato y universitarios de Ciencias Sociales y de la Salud, con lo cual, los resultados obtenidos podrían complicar la extrapolación a la población juvenil. Por otro lado, el estudio es de tipo transversal, de modo que refiere dificultad para establecer valores basales para su comparación entre poblaciones y periodos de tiempo, entre otras. Asimismo, debido a la sensibilidad del tema objeto de estudio puede suponerse cierto sesgo de deseabilidad social.

Por último, dada la incidencia que tiene el consumo de alcohol de los jóvenes en las emergencias sociosanitarias es indispensable continuar con esta línea de investigación, identificando patrones y trastornos de consumo en este sector de población y proponiendo estrategias de prevención, dado el consumo intensivo de alcohol en población juvenil asociado con politoxiconsumo, tanto en España (Delegación del Gobierno para el Plan Nacional sobre Drogas (2015), como en México (Gómez-Maqueo, Gómez, Morales, \& Pérez, 2009). Ello es relevante si se considera que es un importante factor de riesgo para el abuso/dependencia alcohólica (Balodis, Potenza, \& Olmstead, 2009; García, Novalbos, Martínez, \& O'Ferrall, 2016).

\section{Conflicto de intereses}

Los autores declaran no tener conflictos de interés en relación al presente artículo. 


\section{REFERENCIAS}

Arias, R., Ruiz, R., Fernández, J.A., Pérula, L.A., Muriel, M., \& Márquez, E. (2005). Validación del cuestionario AUDIT para la identificación del consumo de riesgo y de los trastornos por el uso de alcohol en mujeres. Atención Primaria, 36(9), 499-506. http://dx.doi.org/10.1016/S0212-6567(05)70552-7

Balodis, I.M., Potenza, M.N., \& Olmstead, M.C. (2009). Binge drinking in undergraduates: relationships with sex, drinking behaviours, impulsivity, and the perceived effects of alcohol. Behavioural Pharmacology, 20, 518-26. http://dx.doi.org/10.1097/FBP.0b013e328330c779

Carlson, S.R., Johnson, S.C., \& Jacobs, P.C. (2009). Disinhibited characteristics and binge drinking among university student drinkers. Addictive Behaviors, 35, 242-51. http://dx.doi.org/10.1016/j.addbeh.2009.10.020

Clemente, C., Echarte, J.L., Aguirre, A., Puente, I., Iglesias, M.L., \& Supervía, A. (2011). Diferencias en las intoxicaciones de los españoles y los extranjeros atendidas en urgencias. Emergencias, 23, 271-75.

Contel, M., Gual, A., \& Farran, J. (1999). Test para la identificación de trastornos por uso del alcohol (AUDIT): Traducción y validación del AUDIT al catalán y al castellano. Adicciones, 11(4), 337-47. http://dx.doi.org/10.20882/adicciones.613

Delegación del Gobierno para el Plan Nacional sobre Drogas (2014). Encuesta sobre Uso de Drogas en Estudiantes de Enseñanzas Secundarias (ESTUDES) 2012-2013. Ministerio de Sanidad, Servicios Sociales e Igualdad. Gobierno de España. (consultado el 11-022015). Disponible www.pnsd.msssi.gob.es/noticiasEventos/notas/2014/home.htm

Delegación del Gobierno para el Plan Nacional sobre Drogas. Observatorio Español de la Droga y las Toxicomanías (2015). Encuesta Domiciliaria sobre Alcohol y otras Drogas (EDADES) 2013/2014. Ministerio de Sanidad, Servicios Sociales e Igualdad. Gobierno de España (consultado 11-03-2016) Disponible en www.msssi.gob.es/gabinete/notasPrensa.do?id=3581

Díaz, A., Díaz, L.R., Hernández-Ávila, C.A., Narro, J., Fernández, H., \& Solís, C. (2008). Prevalencia del consumo riesgoso y dañino de alcohol y factores de riesgo en estudiantes universitarios de primer ingreso. Salud Mental, 31, 271-82. http://dx.doi.org/10.1590/S0036$\underline{36342002000200005}$

Facundo, F.R., Pedrão, L.J., Aguilar, L., García, K., \& Almanza, E. (2007). Trastornos por consumo de alcohol (AUDIT) en adolescentes y jóvenes marginales de bandas juveniles de México. Rev Enfermagem, 11(4), 611-18. http://dx.doi.org/10.1590/S1414$\underline{81452007000400009}$

Galicia, M., Alonso, J.R., \& Nogué, S. (2014). Intoxicaciones por drogas de abuso: substancias emergentes en el siglo XXI. Emergencias, 26, 47280.

García, M.A., Novalbos, J.P., Martínez, J.M., \& O’Ferrall, C. (2016). Validación del test para la identificación de trastornos por uso de alcohol en población universitaria: AUDIT y AUDIT-C. Adicciones, 28(3), 123-13. http://dx.doi.org/10.20882/adicciones.775
Gómez-Maqueo, E., Gómez, H.L., Morales, B., \& Pérez, M. (2009). Uso del AUDIT y el DAST-10 para la identificación de abuso de sustancias psicoactivas y alcohol en adolescentes. Revista Colombiana de Psicología, 18(1), 9-17.

Guitart, A.M., Espelt, A., Castellano, Y., Bartroli, M., Villalbí, J.R., DomingoSalvany, A., \& Brugal, M.T. (2011). Impacto del trastorno por consumo de alcohol en la mortalidad: ¿hay diferencias según la edad y el sexo?. Gaceta Sanitaria, 25(5), 385-90. http://dx.doi.org/10.1016/j.gaceta.2011.03.019

Isorna, M., Fariña, F., Sierra, J.C., \& Vallejo, P. (2015). Binge drinking: conductas sexuales de riesgo y drogas facilitadoras del asalto sexual en jóvenes españoles. Summa Psicológica, 22(1), 1-8.

Luque, L.E., Gómez, R.A., Cortés, M., Espejo, B., \& Giménez, J.A. (2014). Consumo de alcohol en atracón en jóvenes: evaluación de un instrumento basado en la Teoría de la Conducta Planificada. Revista Argentina de Ciencias del Comportamiento, 6(2), 65-74.

Medina-Mora, E., Carreño, S., \& De la Fuente, R. (1998). Experience with the Alcohol Use Disorders Identification Test (AUDIT) in Mexico. En M. Galanter (editor): Recent Developments in Alcoholism. Volume 14, The Consequences of Alcoholism. Pp 384-396). New York: Plenum Press.

Moral, M.V. \& Ovejero, A. (2011). Consumo abusivo de alcohol en adolescentes españoles: tendencias emergentes y percepciones de riesgo. Universitas Psychologica, 1, 71-87.

Morales, L.A., Bringas, C., Moral, M.V., Rodríguez, F.J., Cetz, C., \& Arriaga, L.O. (2015). Actitudes de permisividad y resistencia, motivaciones y percepciones de riesgo ante el consumo de alcohol en estudiantes mexicanos. Revista Española de Drogodependencias, 40(4), 12-29.

Motos, P., Cortés, M.T., Giménez, J.A., \& Cadaveira, F. (2015). Predictores del consumo semanal de alcohol y sus consecuencias asociadas en universitarios consumidores intensivos de alcohol. Adicciones, 27(2), 119-31. http://dx.doi.org/10.20882/adicciones.700

Organización Mundial de la Salud (OMS) (1992). Programa sobre abuso de sustancias. AUDIT: Prueba de Identificación de Trastornos Derivados del Consumo de Alcohol: Pautas de uso en la atención primaria de la salud (consultado 01-04-2016). OMS/PAS/92.4. Disponible en www.who.int/substance_abuse/activities/en/AUDITmanualSpanish

Sánchez, A., Redondo, S., García, M.I., \& Velázquez, A. (2012). Episodios de urgencia hospitalaria relacionados con el consumo de alcohol en personas de entre 10 y 30 años en Castilla y León durante e período 2003-2010. Revista Española de Salud Pública, 86(4), 409-17.

Sanz, N., Arias, V., Trenchs, V., Curcoy, A., Matalí, J.L., \& Luaces, C. (2009). Consultas por intoxicación etílica aguda en un servicio de urgencias pediátricas. Anales de Pediatría, 7(2), 132-36. http://dx.doi.org/10.1016/j.anpedi.2008.10.006

Saunders, J.B., Aasland, O.G., Babor, T., De la Fuente, J.R., \& Grant, M. (1993). Development of the Alcohol Use Disorders Identification Test (AUDIT). Who collaborative project on early detection of persons with harmful alcohol consumption. Addiction, 88, 791-804 http://dx.doi.org/10.1111/j.1360-0443.1993.tb02093.x 
Secretaría de Salud, Subsecretaría de Prevención y Promoción de la Salud y Dirección General de Epidemiología. Sistema de Vigilancia Epidemiológica de las Adicciones - SISVEA- (2013). Informe 2011 (consultado 25-03-2015). Disponible en http://www.epidemiologia.salud.gob.

Villar, M., Pinto, S., Barizon, L., \& Ferreira, J.L. (2016). Síndrome de dependencia alcohólica en servicios de urgencia: protocolo de evaluación para la práctica profesional de enfermería. Enfermería Global, 15, 78-120. http://hdl.handle.net/10201/47413 